\title{
Prognostic Factors and Outcomes for Patients With Myxofibrosarcoma: A 13-Year Retrospective Evaluation
}

\author{
HEINRICH M.L. MÜHLHOFER ${ }^{1}$, ULRICH LENZE ${ }^{1}$, ALEXANDRA GERSING ${ }^{2}$, VINCENT LALLINGER ${ }^{1}$, \\ RAINER BURGKART ${ }^{1}$, ANDREAS OBERMEIER ${ }^{1}$, RÜDIGER VON EISENHART-ROTHE ${ }^{1}$ and CAROLIN KNEBEL ${ }^{1}$ \\ ${ }^{1}$ Department of Orthopaedic Surgery, Rechts der Isar Hospital, Technical University of Munich, Munich, Germany; \\ ${ }^{2}$ Department of Radiology, Rechts der Isar Hospital, Technical University of Munich, Munich, Germany
}

\begin{abstract}
Background: Soft-tissue sarcomas are rare entities that are divided into approximately 50 histological subtypes. Myxofibrosarcoma (MFS) represents approximately $20 \%$ of all soft-tissue sarcomas, especially in elderly patients in their sixth to eighth decades of life. The treatment for soft-tissue sarcomas varies from primary surgical resection to neoadjuvant or adjuvant radiotherapy or cytotoxic chemotherapy. The aim of this study was to evaluate the prognostic factors affecting survival of patients with MFS, taking into account gender, tumour grade, state of the resection margin, local recurrence, use of radiotherapy, presence of metastases and blood levels of haemoglobin and $C$-reactive protein in a retrospective, single-centre analysis with a minimum follow-up period of 60 months (range=60-156 months). Patients and Methods: The study included 34 patients (malelfemale=20/14). Tumour localization, tumour grade, tumour margins, local recurrence, the use of radiotherapy, the presence of metastasis, and the blood levels of haemoglobin and C-reactive protein preoperatively and during follow-up were evaluated. Results: MFS constituted the most common high-grade sarcoma $(G 2 / G 3,79.4 \%)$ in our cohort and was generally located in a lower limb (73.6\%). Negative margins $(R 0)$ were detected in $67.6 \%$ of patients after surgical resection, and local recurrence occurred in $23.5 \%$ of all patients after a mean disease-free period of 19.4 months. Both parameters exerted no significant influence on survival. Radiotherapy was performed in a neoadjuvant or an adjuvant setting in 50\% of patients (eight neoadjuvant, nine adjuvant). Metastasis occurred after a mean of 20.4 months in $38.2 \%$ of the patients. Higher C-reactive protein levels showed a trend towards being associated with
\end{abstract}

Correspondence to: Heinrich ML Mühlhofer, MD, Department of Orthopaedic Surgery, Ismaninger Str. 22, 81675 Munich, Germany. Tel: +49 8941405490, Fax: +49 8941404849, e-mail: heinrich. muehlhofer@mri.tum.de

Key Words: Myxofibrosarcoma, prognostic factors, outcome, survival. worse survival, but the association was not significant ( $p=0.084$ ); haemoglobin level had no influence on the survival rate ( $p=0.426)$. Tumour grade and metastasis were significant prognostic factors of survival (log-rank test $p=0.041$ and $p=0.00007)$. Ten patients (29.4\%) died due to MFS during our follow-up period. Conclusion: The tumour grade and metastasis of MFS are independently associated with diseasespecific survival, whereas negative surgical margins, local recurrence and blood levels of $C$-reactive protein and haemoglobin were not significant prognostic factors. The understanding of the molecular biological patterns that result in the metastasis of these tumours will help develop better treatment plans in the future.

Soft-tissue sarcoma is a rare disease with an annual incidence of approximately 2-5 per 100,000 per year (1). Divided into approximately 50 histological subtypes, it represents a very heterogenic group of tumour entities. Therefore, the treatment for soft-tissue sarcoma varies from primary surgical resection to neoadjuvant/adjuvant cytotoxic chemotherapy. Notwithstanding the availability of treatment, nearly 5,000 patients die as a consequence of this disease in the U.S. each year, with approximately 12,000 new diagnoses made annually (2). Among the wide variety of soft-tissue sarcomas, one of the most common found in elderly people is myxofibrosarcoma (MFS), representing approximately $20 \%$ of all soft-tissue sarcomas (3). First described in the 1970s by Angervall et al. and specified as an independent disease by the World Health Organization (WHO) in 2002, MFS holds a special position as a difficult-to-treat tumour among the 50 subtypes (4) because it shows different growth patterns, resulting in a strong tendency for local recurrence (5).

The gold-standard treatment for MFS is surgical resection with histologically negative margins, which has superior results and good clinical outcomes compared to exclusive radiotherapy/chemotherapy $(6,7)$. However, negative margins are sometimes difficult to obtain because of the unusual growth patterns along fascial planes with infiltrative activity, which is described as a tail-like growth pattern $(5,8-11)$. This 
difficulty in obtaining clean margins results in higher recurrence rates than other soft-tissue sarcomas, reaching up to $79 \%(8,12)$. The second problem is the tendency for metastasis to the lungs and lymph nodes (6). Recent studies have examined prognostic factors, aiming to identify patients with a major risk of treatment failure (7). Previous studies reported small cohorts with magnetic resonance imagingdiagnostic criteria or histopathological expression of different biomarkers. Our cohort represents a large number of patients with MFS with extensive clinical patient records.

Therefore, the aim of this study was to determine the prognostic factors influencing the survival of patients affected by MFS taking into account the tumour grade, histological subtype, state of resection, size, location, metastases and recurrence in a retrospective, single-centre analysis over the course of 13 years.

\section{Patients and Methods}

We retrospectively reviewed our institution's database to identify patients who underwent treatment for the diagnosis of MFS from January 2005 to August 2016. We identified 53 patients (male/female $=32 / 21)$ with a median age of 65.5 years (range $=26-94$ years) at the time of diagnosis of MFS (Figure 1). All patients were primarily treated with resection at our Institution. We recorded the age (at the time of diagnosis), gender, localization, grade (G1, G2 and G3), tumour margin status (R0: negative/clear margins, R1: positive/involved margins (microscopic), R2: positive/involved margins (macroscopic), Rx: the presence of residual tumour cannot be assessed), the use of radiation therapy (neoadjuvant, adjuvant), the use of chemotherapy, survival, recurrence, metastases (location, time of occurrence), laboratory values (leucocyte count, C-reactive protein (CRP) level, and haemoglobin level) and duration of follow-up (months from diagnosis). Survivorship analysis was performed using the Kaplan-Meier method. Prognostic factors and their influence on mortality were determined using the log-rank test (Mantel-Cox). All data are reported as the means, standard deviations, and percentages, where applicable. The minimum follow-up period for 53 patients was 24 months (range=24-156 months); for 34 patients (male/female $=20 / 14$ ), the minimum follow-up period was 60 months (range $=60-156$ months, median=78 months). Statistical analysis was performed using SPSS 22.0 (IBM, Armonk, NY, USA).

\section{Results}

Overall, we determined that there were 25 tumours $(73.6 \%)$ in 18 patients $(54.1 \%)$ located in the lower limb, especially in the thigh, followed by the upper limb in eight patients (23.6\%, Figure 2). The overall survival considering all 53 patients was $81.1 \%$ at 24 months after the initial diagnosis, and the overall survival of the 34 patients with a minimum follow-up period of 60 months was $70.6 \%$ (Figure 3 ). In our study, the survival of females was better than that of males, but this difference was not statistically significant (Figure 4; $\log$-rank test $p=0.197)$. Concerning the tumour grade in the 34 patients with the longer follow-up period, there were seven (20.6\%) G1 tumours, 14 (41.2\%) G2 tumours, and 13 (38.2\%) G3 tumours. The tumour grade significantly positively correlated (log-rank test $p=0.041$ ) with patient survival, with those with G1 tumours having best survival (Figure 5). All patients underwent surgical resection. Negative margins (R0) were detected in 23 out of the 34 patients $(67.6 \%)$, five $(14.7 \%)$ were classified as R1, one as R2 $(2.9 \%)$, and two as Rx $(5.9 \%)$. Three patients had no available information about the resection margins. Compared to patients with $\mathrm{R} 0$ resection margins, patients with $\mathrm{R} 1$ resection margins did not have poorer survival (Figure 6). Seventeen patients $(50 \%)$ underwent radiation (neoadjuvant in eight, adjuvant in nine, Figure 7). All patients who underwent radiation had G2 or G3 tumours (high-grade sarcoma). There were no significant differences in the rates of survival among patients who received neoadjuvant radiotherapy, adjuvant radiotherapy and no radiotherapy (log-rank test $p=0.055)$. No patient underwent chemotherapy.

Local recurrence was detected in eight patients $(23.5 \%)$, but local recurrence had no effect on overall survival $(p=0.757$, Figure 8). The mean time to local recurrence was 19.4 months. We observed metastases in $13(38.2 \%)$ out of the 34 patients. The occurrence of metastasis significantly negatively correlated (log-rank test $p=0.00007$ ) with patient survival. The mean time to the first appearance of metastasis was 20.5 months. The survival of patients with metastases is illustrated in Figure 9. Serum parameters were analysed preoperatively the day before resection of MFS. A high CRP level demonstrated a tendency to be associated with worse survival, but without statistical significance (log-rank test $p=0.084$, Figure 10). The haemoglobin level was also measured preoperatively and had no association with the survival outcome (log-rank test $p=0.426$, Figure 11). Ten patients (29.4\%) died due to MFS during our follow-up period. The survival rate was significantly affected by the tumour grade and presence of metastases (Figures 3, 5 and 9). Patients with metastases had significantly worse overall survival than patients without metastases (hazard ratio $=2.97, p=0.005$ ) and than patients with G3 MFS (hazard ratio=1.56, $p=0.024$ ).

\section{Discussion}

In the current study, our working group retrospectively analysed a single-centre cohort treated at a national sarcoma centre; members of the cohort had a history of MFS and a median follow-up period of 78 months. We sought to determine the prognostic factors and their influence on mortality, recurrence and metastasis. MFS represents approximately $20 \%$ of all sarcomas in adults and is known for unique histopathologic characteristics and growth patterns, such as the lack of a pseudo-capsule limiting tumour growth and the tendency to spreading along fascial layers, making it known as a difficult to treat lesion among 


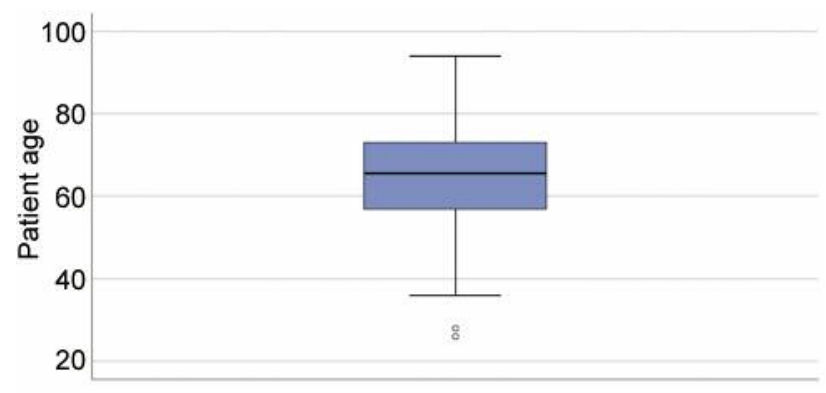

Figure 1. Age of distribution of study collective.

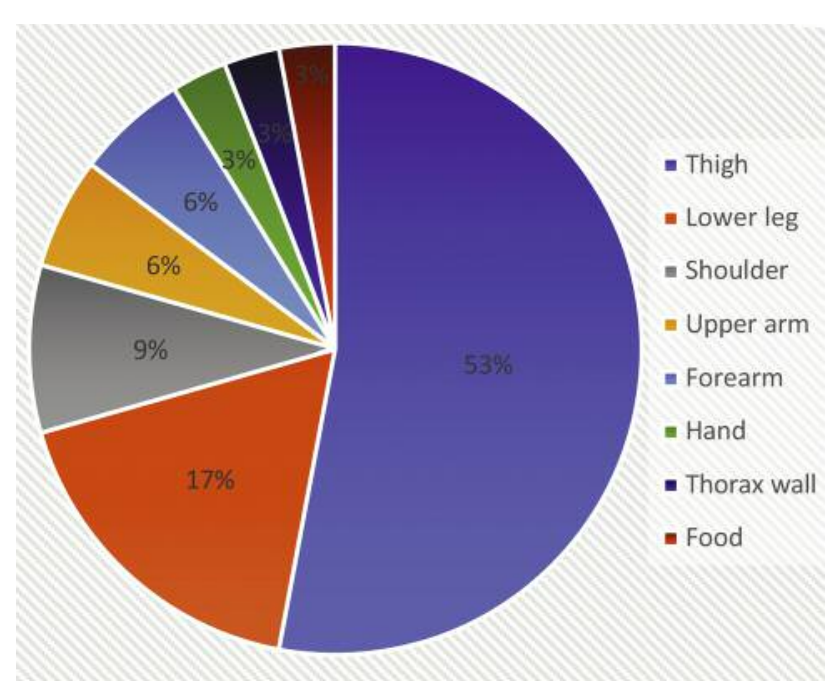

Figure 2. Frequency of tumour localisation.

tumour surgeons $(3,8)$. The survival rate was highly dependent on the tumour grade, the achievement of negative margins during surgery, and metastasis. Additionally, certain serological markers were found to be prognostic factors.

To the best of our knowledge, this is the most up-to-date report providing a comprehensive epidemiological and prognostic evaluation of MFS in a large population treated at a specialized centre.

Localization and overall survival. In the present study, the age distribution of patients suffering MFS was comparable to that in the existing literature. Our cohort was predominantly male, with a median age of 65.5 years, which is similar to the cohorts of Kikuta et al. (8), Look Hong et al. (10) and Dewan et al. (9). The most common tumour site was the lower extremity, with $60 \%$ of the tumours in this study found in the thigh, followed by the lower leg and upper arm, confirming the findings of

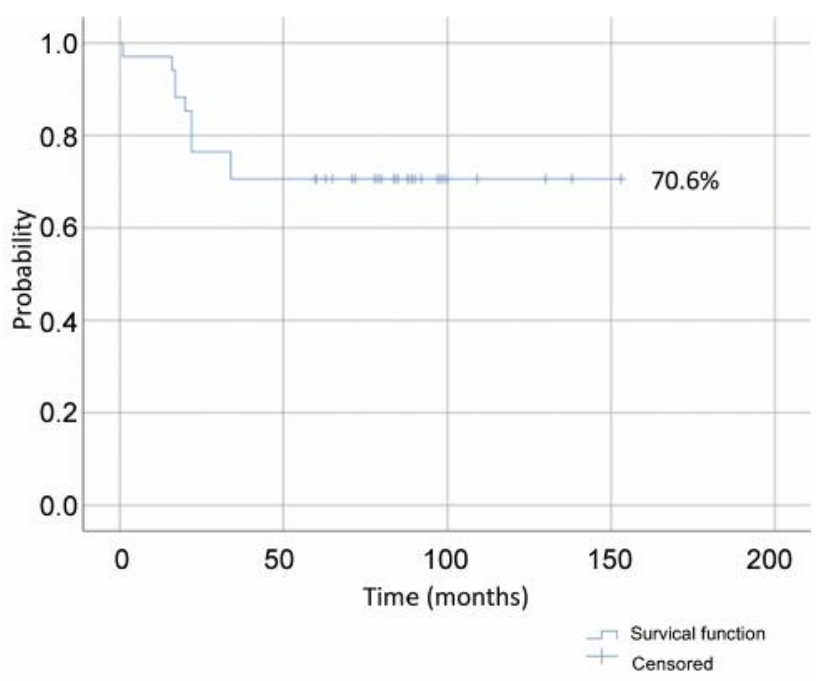

Figure 3. Kaplan-Meier curve showing statistical analysis of overall survival in months after initial diagnosis of myxofibrosarcoma. Minimum follow-up of 60 months, 34 patients.

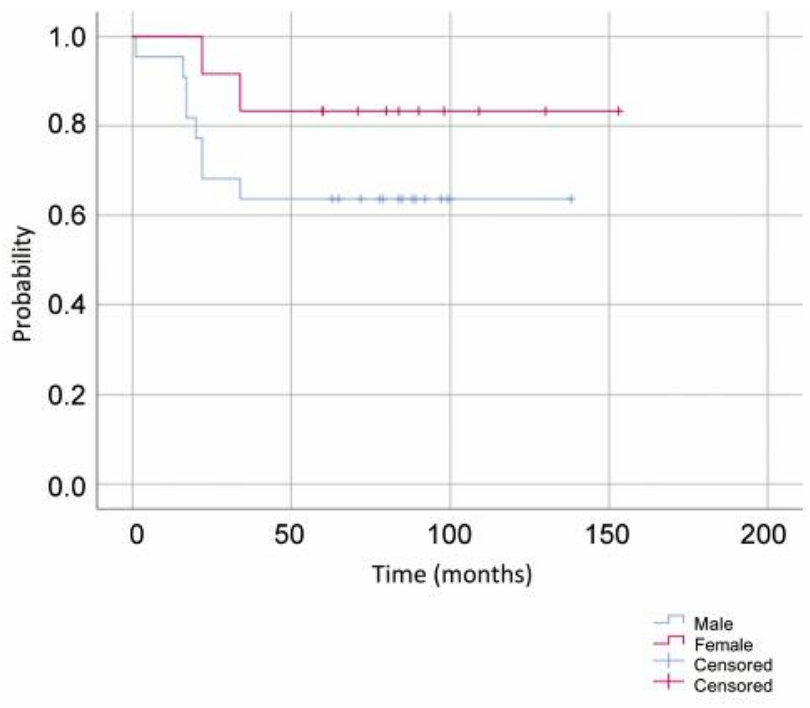

Figure 4. Kaplan-Meier curve showing statistical analysis of survival in months after initial diagnosis according to gender; log-rank $p=0.197$. Minimum follow-up of 60 months, 34 patients.

Daniels et al. (11) and Sambri et al. (12). We found an overall 5 -year survival rate of $70.6 \%$, which was slightly lower than the $80 \%$ found by Sanfillipo et al. (5). Dewan et al. found a 5-year survival rate of $63 \%$ but included mostly patients with highgrade (G3) tumours in their cohort (9). In our cohort, the overall survival rates did not significantly correlate with the location of 

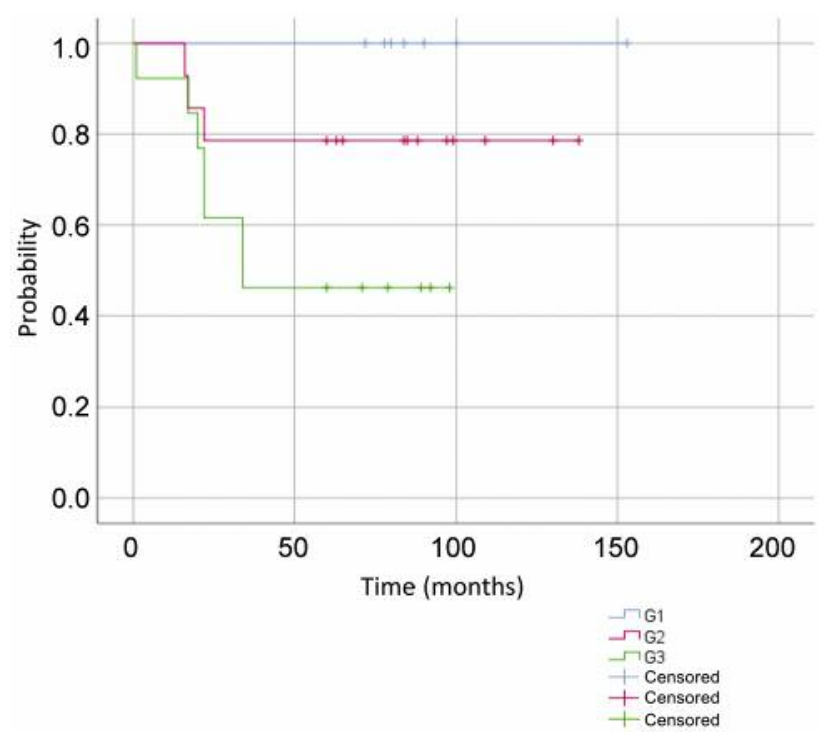

Figure 5. Kaplan-Meier curve showing statistical analysis of survival in months after initial diagnosis according to grading of myxofibrosarcoma; log-rank $p=0.041$. Minimum follow-up of 60 months, 34 patients.

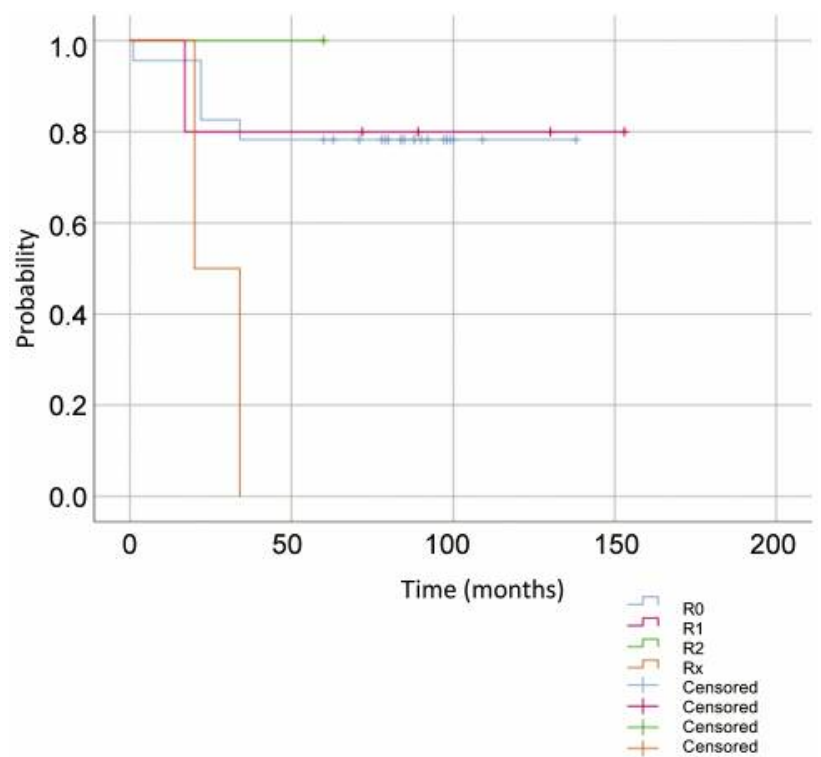

Figure 6. Kaplan-Meier curve showing statistical analysis of survival in months after initial diagnosis according to resection margins of myxofibrosarcoma; log-rank $p=0.058$. Minimum follow-up of 60 months, 34 patients.

the MFS or the gender of the patients, although we detected a trend towards a slightly higher survival rate for female than for male patients (see Figure 4). In the literature, the survival rate of female patients seems to be slightly better than that of male

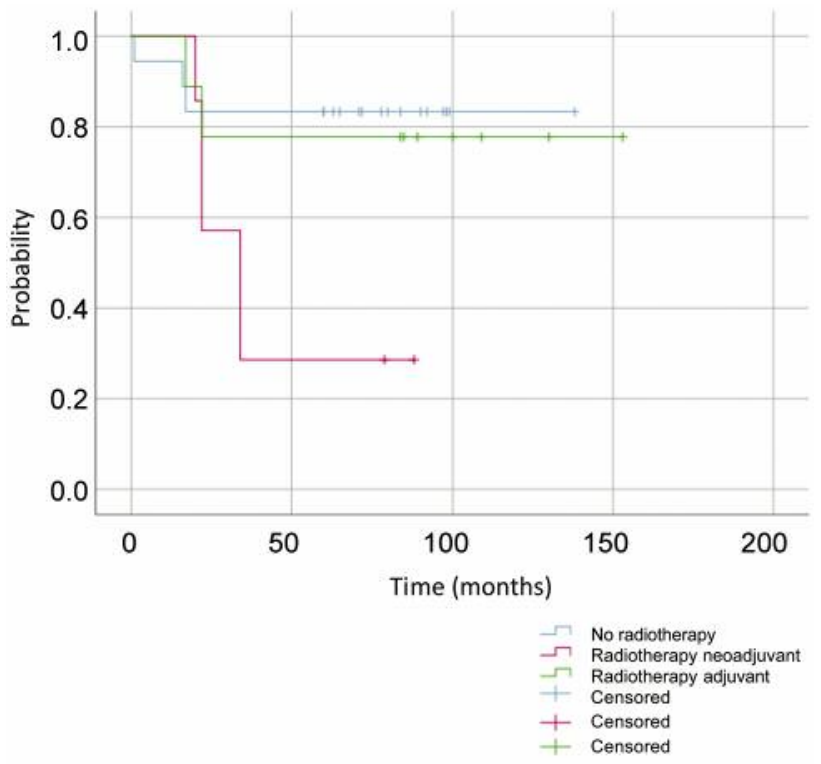

Figure 7. Kaplan-Meier curve showing statistical analysis of survival in months after initial diagnosis according to radiotherapy of myxofibrosarcoma; log-rank $p=0.055$. Minimum follow-up of 60 months, 34 patients.

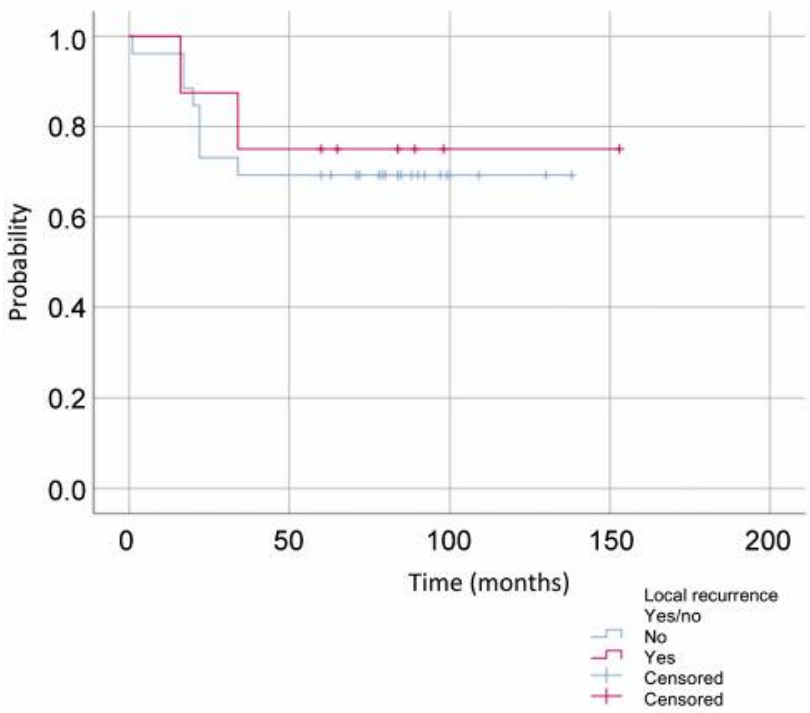

Figure 8. Kaplan-Meier curve showing statistical analysis of survival in months after initial diagnosis according to local recurrence of myxofibrosarcoma; log-rank $p=0.757$. Minimum follow-up of 60 months, 34 patients.

patients, although the underlying reasons remain unclear. We confirmed the results of Abbott et al. who found a better survival rate for females with retroperitoneal sarcoma than males in their cohort of 1199 patients (13). 


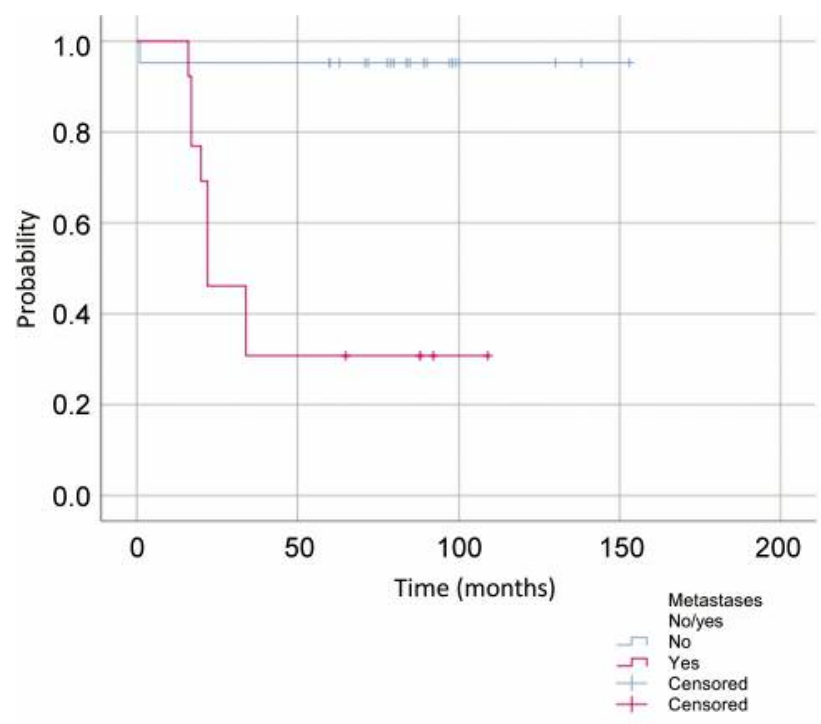

Figure 9. Kaplan-Meier curve showing statistical analysis of survival in months after initial diagnosis according to metastasis of myxofibrosarcoma; log-rank $p=0.00007$. Minimum follow-up of 60 months, 34 patients.

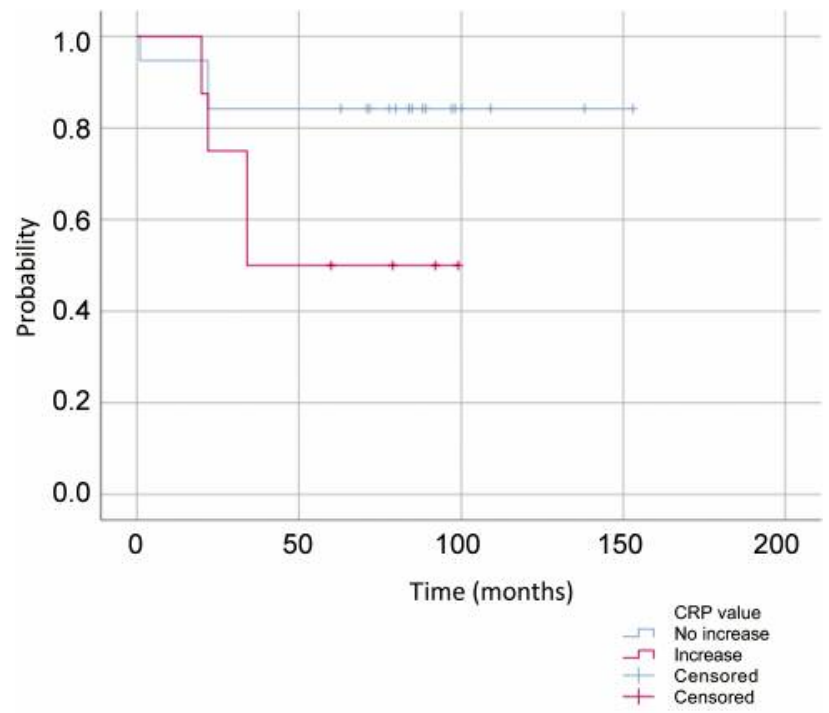

Figure 10. Kaplan-Meier curve showing statistical analysis of survival in months after initial diagnosis according to serum level of $C$-reactive protein (CRP) before operation; log-rank $p=0.084$, cut-off $=0.5 \mathrm{mg} / \mathrm{dl}$. Minimum follow-up of 60 months, 34 patients.

Grading. In terms of histopathology, most of the patients in our cohort were specified as having high-grade MFS (34 patients), in addition to 14 with G2 MFS and 13 with G3 MFS. The proportion of patients with G3 tumours was slightly higher than that reported by Sanfilipo et al. (5). Nevertheless, the low incidence of G1 tumours confirmed the findings of

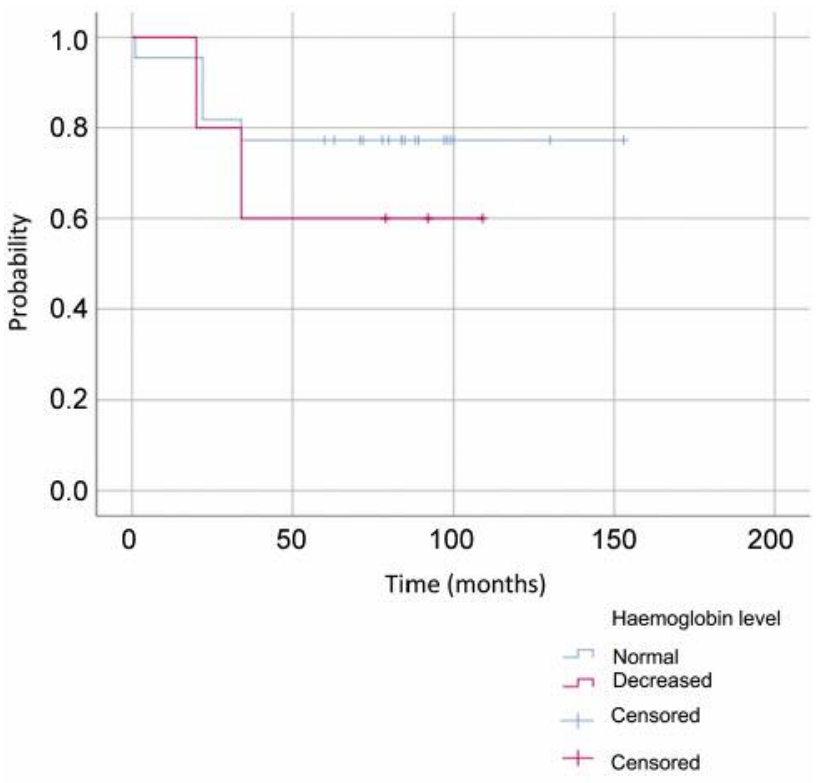

Figure 11. Kaplan-Meier curve showing statistical analysis of survival in months after initial diagnosis according to haemoglobin level before operation; log-rank $p=0.426$, cut-off $<13 \mathrm{~g} / \mathrm{dl}$ for males, $<12 \mathrm{~g} / \mathrm{dl}$ for females. Minimum follow-up of 60 months, 34 patients.

Dewan et al. (10) and Sanfilippo et al. (5). No patients with low-grade G1 MFS died of the disease in our cohort, confirming that grading is an important prognostic factor. Patients who had high-grade G3 MFS had the worst 5-year survival rates among patients with different grade tumours in our study. Compared to the $73.2 \%$ survival rate reported by Sambri et al. (12), our survival rate of $46.2 \%$ was significantly lower. Our 5-year survival rate was lower than those found by other working groups, with 5-year survival rates ranging from 55 to $63 \%$. Patients with G2 tumours had a significantly better survival rate than those with G3 tumours, which is in agreement with the findings of the existing literature.

Negative margins and local recurrence. Negative margins and local recurrence are important considerations in the treatment of MFS. Kikuta et al. described the unique histological growth patterns of MFS in their cohort of 30 patients with recurrence of MFS. Histologically negative margins were achieved in only $40 \%$ of their cohort (8). This working group clearly showed the relationship between positive margins after the first surgery and the incidence of local recurrence. The unique growth patterns of MFS are most likely responsible for this. These special growth patterns are even visible on the preoperative MRI and are known as tail signs, representing fascial enhancement extending from the tumour margin (14). In our series, we achieved negative margins in $67.6 \%$ (23 patients), with positive margins in six patients. These proportions are 
slightly lower than those in the cohort of Kikuta et al. (8). However, the cohorts are not directly equivalent, as Kikuta et al. included amputees in their negative margins subgroup. We excluded patients who underwent amputation because limb salvage was one of our primary treatment goals. We confirmed the results of the large series by Mutter et al., who also excluded patients who underwent amputation from their cohort (15). Nevertheless, we must highlight the issue of the definition of negative margins because it is not standardized. Common definitions of negative margins range between 1 $\mathrm{mm}$ and $5 \mathrm{~cm}$; some patients may be classified as having negative margins by one author and as having positive margins by another $(5,8)$. The recent work of Harati et al. showed that a tumour-free margin $>1 \mathrm{~mm}$ is clinically relevant (20), which could help define a standard to be applied in a meta-analysis of a large number of cohorts in the future. We analysed resection margins by gross and microscopic examinations and then defined a clear tumourfree margin of at least $1 \mathrm{~mm}$ from the edge of the inked specimen. As described in recent studies, a negative margin can be defined as $>1 \mathrm{~mm}$ in MFS and in other soft-tissue sarcoma (16-18). We calculated a 5-year survival rate of $78.3 \%$ for patients with negative margins. Surprisingly, the 5-year survival rate for patients with R1 positive margins was slightly but not significantly higher than that of patients with negative margins. This finding could be the result of statistical bias, as only one out of the 13 patients with positive margins (R1) died during the follow-up period. We have no other explanation. The worst survival rate was found for patients with Rx positive margins; these findings reflect observations in previous studies and are comparable with observations in studies of other soft-tissue sarcomas (19).

In our cohort, 10 patients developed local recurrence in the follow-up period. All of these patients had G2 (3) or G3 (7) tumours. Local recurrence occurred independently of the state of the margins; in fact, most patients with local recurrence had histologically negative margins. We detected a worse 5-year survival rate in the group with local recurrence than in that without local recurrence, although the difference between these groups was not significant. Thirteen patients also developed distant metastases. Local recurrence is a major problem for surgeons and patients. Reoperation is much more difficult due to neurovascular structures and scar tissue because most MFSs are located deep in the thigh. This fact was examined by Kikuta et al. in their cohort of patients with local recurrence, who had a 5-year second recurrence-free survival rate of $9.8 \%$ (8).

Distant metastases. Thirteen patients (38.2\%) developed distant metastases during the study period. The average time to appearance of distant metastases was 20.5 months. The detected metastases were located mainly in the lung $(n=12)$ and the lymph nodes $(n=2)$. We detected one in the gluteal muscles, one in the periossar and one in a vertebra. The rate of development of metastases varies in the literature from $9.5-27.5 \%(5,6,9,10,15,20,21)$. In our cohort, the rate of metastasis was equivalent to the highest reported in the literature; therefore, we confirmed the findings of Tsuchie et al., who also reported a comparable rate of metastases (6). All other authors reported rates of metastasis between 9.3 and $23 \%$. We have no explanation for these findings; we can only surmise that our tumour cohort had different mitotic activity, which is a known risk factor for distant metastases, and different tumour-specific characteristics, such as the presence of necrosis $(5,22,23)$. The tumour sites in our cohort match those in recent reports in the literature. However, from a prognostic point of view, we found a clear correlation between metastasis and poorer survival. Patients who developed distant metastases after surgery had a 5-year survival rate of $30.8 \%$ while those who did not had a 5 -year survival rate of $95.2 \%$.

Serum analysis. Recent studies have addressed the effect of systemic inflammation on disease progression and metastases in patients with cancer. Only a few studies have addressed this problem in those with soft-tissue sarcomas $(24,25)$. An elevated preoperative CRP level was discussed as an independent risk factor for poor survival (26). Panotopoulos et al. confirmed this in their cohort of patients with liposarcoma and postulated that the level of CRP is a predictor of disease-specific survival in patients with liposarcoma (27). Moreover, preoperative anaemia is known to be associated with shorter 5-year survival rates in patients with cancer, according to the European Cancer Anaemia Survey (28). Nakamura et al. noted that anaemia was correlated with poor survival rates in patients with soft-tissue sarcomas (29). To the best of our knowledge, our working group is the first to determine the correlation between the serum level of CRP and survival rates in a cohort of patients with MFS. We did not find any significant correlation between decreased values of haemoglobin and poor survival. A low haemoglobin level is a positive indicator for anaemia; therefore, we confirmed the results of Nakamura et al. (32). They reported a 5-year survival rate of $52.6 \%$ for patients with anaemia and $79.7 \%$ for those without anaemia. We found similar percentages of $60.0 \%$ and $77.3 \%$, respectively. Furthermore, we demonstrated an apparent but nonsignificant association between elevated CRP serum level and poor survival $(p=0.084)$. This suggests that we can confirm the findings of Szkandera et al. (26), who demonstrated a worse survival rate in patients with elevated serum CRP level than in those with a normal level. We calculated nearly the same 5-year survival rate as Szkandera (73.4\%) for patients with elevated serum level of CRP (26). The cohort in the study by Szkadera et al. included 304 patients with all types of soft-tissue sarcomas (26). The lack of a significant 
correlation between the serum level of CRP and survival is probably the result of statistical bias due to the limited number of patients included in our study.

Our study has certain limitations. The small number of patients included in the study limits the statistical analysis, and MFS remains a rare disease; therefore, a multicentre study is needed to solve this problem. We included patients from 2005 onwards and did not have full records regarding interesting new molecular serum markers such as procalcitonin and interleukin. Further studies should address these biomarkers to provide more accurate prediction of tumour prognosis after surgery using serum analysis. Finally, our cohort underwent surgery conducted by four different surgeons, which introduces bias regarding their training levels and experience with MFS.

\section{Conclusion}

In conclusion, MFS is challenging for tumour surgeons and oncologists due to its clinical course. Distant metastasis of MFS leads to a poor prognosis. Negative margins after the first surgery are an important finding that can guide the treatment process, emphasizing the need for primary treatment at a referral hospital. New approaches using serum analysis of different biomarkers may be able to guide more precise prediction of survival in the future. However, understanding the molecular biological patterns that result in growth, recurrence and metastasis of these tumour entities will help develop better treatment plans in the future.

\section{Authors' Contributions}

HM, VL and CK performed patient recruitment and clinical investigation. UL, AO, AG, RB and RvER conceived of the study, participated in its design and coordination and helped draft the article. All Authors read and approved the final article.

\section{Conflicts of Interest}

The Authors declare that they have no financial or non-financial competing interests.

\section{IRB Approval}

The Institutional Review Board (IRB) at the Klinikum rechts der Isar approved the study (Faculty of Medicine, Technical University Munich, 441/16).

Each Author certifies that all investigations were conducted in conformity with ethical principles. Written informed consent was obtained from all patients included in the study.

\section{Funding}

This study was partially funded by the Wilhelm-Sander Foundation (Fördernummer: 2009.905.2), which is a charitable, non-profit foundation whose purpose is to promote cancer reasearch.

\section{Consent for Publication}

No individual person's data are published in the present work.

\section{Availability of Data and Materials}

The datasets supporting the conclusions of this article are included within the article. The raw data can be requested from the corresponding Author.

\section{Acknowledgements}

The study was supported by Wilhelm-Sander Foundation (Project number: 20099052), which is a charitable, not for profit foundation for the promotion of cancer research.

\section{References}

1 Siegel RL, Miller KD and Jemal A: Cancer statistics, 2015. CA Cancer J Clin 65: 5-29, 2015. PMID: 25559415. DOI: $10.3322 /$ caac 21254

2 Stefanovski PD, Bidoli E, De Paoli A, Buonadonna A, Boz G, Libra M, Morassut S, Rossi C, Carbone A and Frustaci S: Prognostic factors in soft tissue sarcomas: a study of 395 patients. Eur J Surg Oncol 28: 153-164, 2002. PMID: 11884051. DOI: 10.1053 /ejso.2001.1242

3 Jo VY and Fletcher CDM: WHO classification of soft tissue tumours: an update based on the 2013 (4th) edition. Pathology 46: 95-104, 2014. PMID: 24378391. DOI: 10.1097/PAT.000000 0000000050

4 Angervall L, Kindblom L-G and Merck C: Myxofibrosarcoma. A study of 30 cases. Acta Pathol Microbiol Scand A 85A: 127140, 1977. PMID: 15396. DOI 10.1111/j.1699-0463.1977. tb00410.x

5 Sanfilippo R, Miceli R, Grosso F, Fiore M, Puma E, Pennacchioli E, Barisella M, Sangalli C, Mariani L, Casali PG and Gronchi A: Myxofibrosarcoma: Prognostic factors and survival in a series of patients treated at a single institution. Ann Surg Oncol 18: 720-725, 2010. PMID: 20878245. DOI 10.1245/s 10434-010-1341-4

6 Tsuchie H, Kaya M, Nagasawa H, Emori M, Murahashi Y, Mizushima E, Myakoshi N, Yamashita T and Shimada Y: Distant metastasis in patients with myxofibrosarcoma. Ups J Med Sci 122: 190-193, 2018. PMID: 28814152. DOI 10.1080/ 03009734.2017 .1356404

7 Scoccianti G, Ranucci V, Frenos F, Greto D, Beltrami G, Capanna R and Franchi A: Soft tissue myxofibrosarcoma: A clinico-pathological analysis of a series of 75 patients with emphasis on the epithelioid variant. J Surg Oncol 114: 50-55, 2016. PMID: 27076198. DOI 10.1002/jso.24250

8 Kikuta K, Nakayama R, Yoshida A, Sasaki A, Kameyama K, Chuman H, Kawai A, Nakamura M, Matsumoto M and Morioka $\mathrm{H}$ : A histological positive margin after surgery is correlated with high local re-recurrence rate in patients with recurrent myxofibrosarcoma. Jpn J Clin Oncol 82: 361-368, 2017. PMID: 28064204. DOI 10.1093/jjco/hyw 199

9 Dewan V, Darbyshire A, Sumathi V, Jeys L and Grimer R: Prognostic and survival factors in myxofibrosarcomas. Sarcoma 2012: 1-5, 2012. PMID: 22736953. DOI 10.1155/2012/830879 
10 Look Hong NJ, Hornicek FJ, Raskin KA, Yoon SS, Szymonifka J, Yeap B, Chen Y-L, DeLaney TF, Nielsen GP and Mullen JT: Prognostic factors and outcomes of patients with myxofibrosarcoma. Ann Surg Oncol 20: 80-86, 2012. PMID: 22890594. DOI 10.1245/s10434-012-2572-3

11 Daniels J, Green CM, Freemont A and Paul A: The management of myxofibrosarcoma - a ten-year experience in a single specialist centre. Acta Orthop Belg 80: 436-441, 2014. PMID: 26280619

12 Sambri A, Bianchi G, Righi A, Ferrari C and Donati D: Surgical margins do not affect prognosis in high grade myxofibrosarcoma. Eur J Surg Oncol 42: 1042-1048, 2016. PMID: 27260849. DOI 10.1016/j.ejso.2016.05.015

13 Abbott AM, Habermann EB, Parsons HM, Tuttle T and AlRefaie W: Prognosis for primary retroperitoneal sarcoma survivors. Cancer 118: 3321-3329, 2012. PMID: 22246900. DOI $10.1002 / \mathrm{cncr} .26665$

14 Yoo HJ, Hong SH, Kang Y, Choi J-Y, Moon KC, Kim H-S, Han I, Yi M and Kang HS: MR imaging of myxofibrosarcoma and undifferentiated sarcoma with emphasis on tail sign; diagnostic and prognostic value. Eur Radiol 24: 1749-1757, 2014. PMID: 24889995. DOI 10.1007/s00330-014-3181-2

15 Mutter RW, Singer S, Zhang Z, Brennan MF and Alektiar KM: The enigma of myxofibrosarcoma of the extremity. Cancer 118 : 518-527, 2011. PMID: 21717447. DOI 10.1002/cncr.26296

16 Gundle KR, Kafchinski L, Gupta S, Griffin AM, Dickson BC, Chung PW, Catton CN, O'Sullivan B, Wunder JS and Ferguson PC: Analysis of margin classification systems for assessing the risk of local recurrence after soft tissue sarcoma resection. J Clin Oncol 36: 704-709, 2018. PMID: 29346043. DOI 10.1093/jac/dkp107

17 Harati K, Kirchhoff P, Behr B, Daigeler A, Goertz O, Hirsch T, Lehnhardt $M$ and Ring A: Soft tissue sarcomas of the distal lower extremities: A single-institutional analysis of the prognostic significance of surgical margins in 120 patients. Oncol Rep 36: 863-870, 2016. PMID: 27278861. DOI 10.3892/ or.2016.4862

18 Ahmad R, Jacobson A, Hornicek F, Haynes AB, Choy E, Cote G, Nielsen GP, Chen Y-L, DeLaney TF and Mullen JT: The width of the surgical margin does not influence outcomes in extremity and truncal soft tissue sarcoma treated with radiotherapy. Oncologist 21: 1269-1276, 2016. PMID: 27440063. DOI 10.1634/theoncologist.2015-0534

19 Knebel C, Lenze U, Pohlig F, Lenze F, Harrasser N, Suren C, Breitenbach J, Rechl H, Eisenhart-Rothe von R and Mühlhofer HML: Prognostic factors and outcome of Liposarcoma patients: a retrospective evaluation over 15 years. BMC Cancer 17: 1-10, 2017. PMID: 28606068. DOI 10.1186/s12885-017-3398-y

20 Kikuta K, Kubota D, Yoshida A, Suzuki Y, Morioka H, Toyama Y, Kobayashi E, Nakatani F, Chuuman H and Kawai A: An analysis of factors related to recurrence of myxofibrosarcoma. Jpn J Clin Oncol 43: 1093-1104, 2013. PMID: 23969617. DOI $10.1093 / \mathrm{jjco} / \mathrm{hyt} 119$

21 Haglund K, Raut C, Nascimento M, Wang Q, George S and Baldini E: Recurrence patterns and survival for patients with intermediate- and high-grade myxofibrosarcoma. Int J Radiat Oncol Biol Phys 82: 361-367, 2012. PMID: 20951504. DOI $10.1016 /$ j.ijrobp.2010.08.042
22 Lin CN, Chou SC, Li C-F, Tsai KB, Chen WC, Hsiung CY, Yen $\mathrm{CF}$ and Huang H-Y: Prognostic factors of myxofibrosarcomas: implications of margin status, tumor necrosis, and mitotic rate on survival. J Surg Oncol 93: 294-303, 2006. PMID: 16496357. DOI $10.1002 /$ jso. 20425

23 Widemann $\mathrm{BC}$ and Italiano A: Biology and management of undifferentiated pleomorphic sarcoma, myxofibrosarcoma, and malignant peripheral nerve sheath tumors: State of the art and perspectives. J Clin Oncol 36: 160-167, 2018. PMID: 29220302. DOI 10.1200/JCO.2017.75.3467

24 Szkandera J, Absenger G, Liegl-Atzwanger B, Pichler M, Stotz M, Samonigg H, Glehr M, Zacherl M, Stojakovic T, Gerger A and Leithner A: Elevated preoperative neutrophil/lymphocyte ratio is associated with poor prognosis in soft-tissue sarcoma patients. Br J Cancer 108: 1677-1683, 2013. PMID: 23558897. DOI 10.1038/bjc.2013.135

25 Nakamura T, Matsumine A, Matsubara T, Asanuma K, Uchida A and Sudo A: The combined use of the neutrophil-lymphocyte ratio and C-reactive protein level as prognostic predictors in adult patients with soft tissue sarcoma. J Surg Oncol 108: 481485, 2013. PMID: 24018883. DOI 10.1002/jso.23424

26 Szkandera J, Gerger A, Liegl-Atzwanger B, Absenger G, Stotz M, Samonigg H, Maurer-Ertl W, Stojakovic T, Ploner F, Leithner $\mathrm{A}$ and Pichler M: Validation of the prognostic relevance of plasma C-reactive protein levels in soft-tissue sarcoma patients. Br J Cancer 109: 2316-2322, 2013. PMID: 24084772. DOI 10.1038/bjc. 2013.595

27 Panotopoulos J, Posch F, Alici B, Funovics P, Stihsen C, Amann G, Brodowicz T, Windhager R and Ay C: Hemoglobin, alkalic phosphatase, and C-reactive protein predict the outcome in patients with liposarcoma. J Orthop Res 33: 765-770, 2015. PMID: 25641201. DOI 10.1002/jor.22827

28 Ludwig H, Van Belle S, Barrett-Lee P, Birgegård G, Bokemeyer C, Gascón P, Kosmidis P, Krzakowski M, Nortier J, Olmi P, Schneider M and Schrijvers D: The European Cancer Anaemia Survey (ECAS): A large, multinational, prospective survey defining the prevalence, incidence, and treatment of anaemia in cancer patients. Eur J Cancer 40: 2293-2306, 2004. PMID: 15454256. DOI 10.1016/j.ejca.2004.06.019

29 Nakamura T, Grimer R, Gaston C, Carter S, Tillman R, Abudu A, Jeys $\mathrm{L}$ and Sudo A: The relationship between pretreatment anaemia and survival in patients with adult soft tissue sarcoma. J Orthop Sci 18: 987-993, 2013. PMID: 23943226. DOI 10.1007/s00776-013-0454-6
Received April 16, 2019

Revised May 11, 2019

Accepted May 16, 2019 